\title{
Novel Silicon Nanowire-based Electron Detector Utilized in Next Generation Scanning Electron Microscopes
}

\author{
Mohammadreza Hajmirzaheydarali ${ }^{1}$, Mehdi Akbari ${ }^{1}$ and Shams Mohajerzadeh ${ }^{1}$ \\ ${ }^{1}$ Nano-Electronics and Thin Film Lab, School of Electrical and Computer Engineering, University of \\ Tehran, Tehran, Iran.
}

Electron microscopes are the scientific tools which utilize highly energetic electron beam (up to $40 \mathrm{keV}$ ) to overcome the light limitations in the study of specimen in $\mathrm{nm}$ scale. Since its invention, Electron microscope has been a valuable tool in the development of scientific theories and it has been contributed in many fields of science such as nanoelectronics, material engineering, biology, medicine and surface engineering [1].

Electron detection has an important role in microscopic analysis and known as one of the most important blocks of SEM. The conventional Everhart-Thornley (E-T) detector utilized optical approach, consists of scintillator, maintained at $12 \mathrm{kV}$ positive potential to attract the incoming electrons from the specimen, light tube, photomultiplier and amplifier [2]. The photons generated due to the interaction of incoming electrons with the scintillator surface travels through a light tube toward the photomultiplier (PMT) block. The signal generated at the output of the detector is due to the light stimulated current which amplified by PMT [3]. Due to optical instruments used in construction of the detector, it is a large sophisticated device which cannot close to the specimen. The positive or negative potential relative to the source of the electrons helps it to attract electrons [4]. For getting more resolution and contrast, it's better to close the detector to the specimen to collect more electrons.

Here, our novel electron detector is a miniaturized nano-structured device which can be approached to the specimen (fig. 1). This detector attracts electrons along with converting their current to voltage directly. The current was measured by a low noise and precise picoammeter. It was fabricated by growing silicon nanowires (SiNWs) on a p-type silicon substrate covered by gold in a VLS process (fig. 2). Gold was used as the catalyst layer for NW growing. The SiNWs with the diameter less than $70 \mathrm{~nm}$ along with Si substrate were highly doped to act as $\mathrm{nm}$ electron sensitive conductive wires (fig $3 \mathrm{a}, 3 \mathrm{~b}$ ). The ultra-low cost, high voltage free, simply fabricated nano-structured detector which eliminates the optical phase from the electron detection process enhances the yield of electron detection in a highly manner.

[1] Goldstein J.I. et al, Scanning Electron Microscopy and X-ray Microanalysis (Third Edition), Kluwer Academic/Plenum Publishers: New York, (2003).

[2] Duncumb, P. and Shields, P.K. In The Electron Microprobe, Wiley: New York, (2003), p. 284.

[3] Richard Brundle Charles C. et al, Encyclopedia of materials characterization, ButterworthHeinemann publications, 1992.

[4] William R. Herguth et al, Applications of Scanning Electron Microscopy and Energy Dispersive Spectroscopy (SEM/EDS) To Practical Tribology Problems. Senior Technical Associate Herguth Laboratories, Inc. 


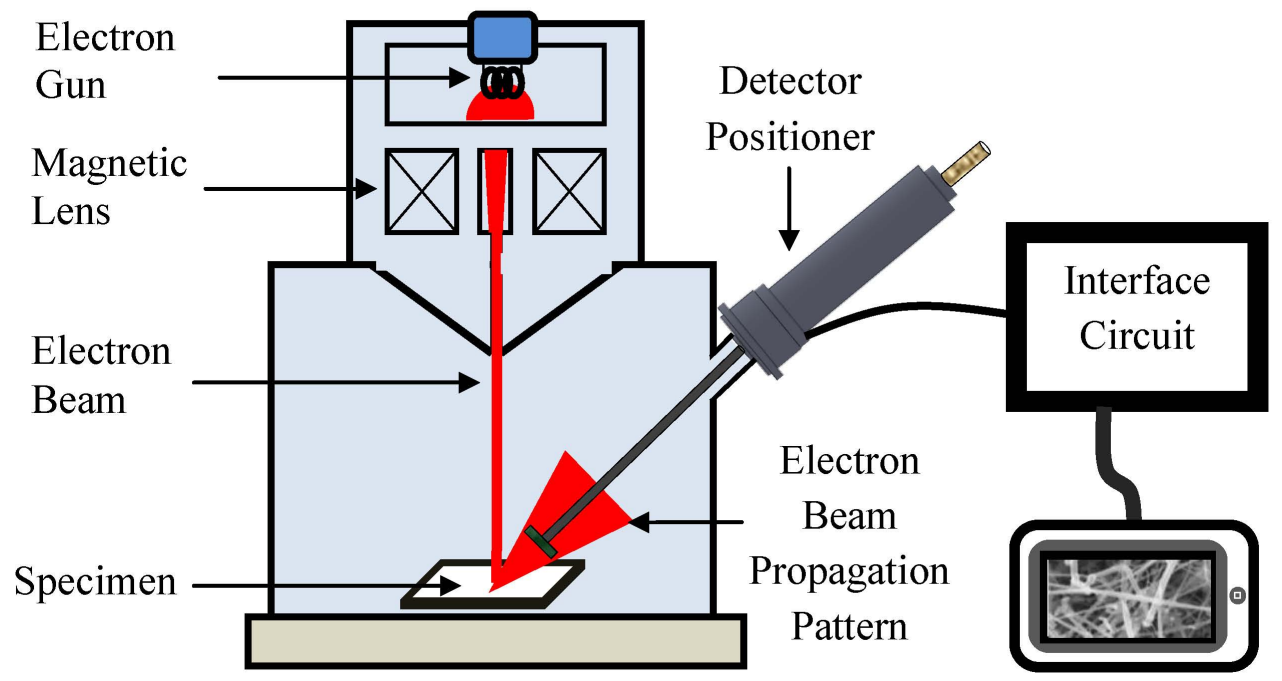

Figure1. Miniaturized nano-structured electron detector located on the detector positioner.

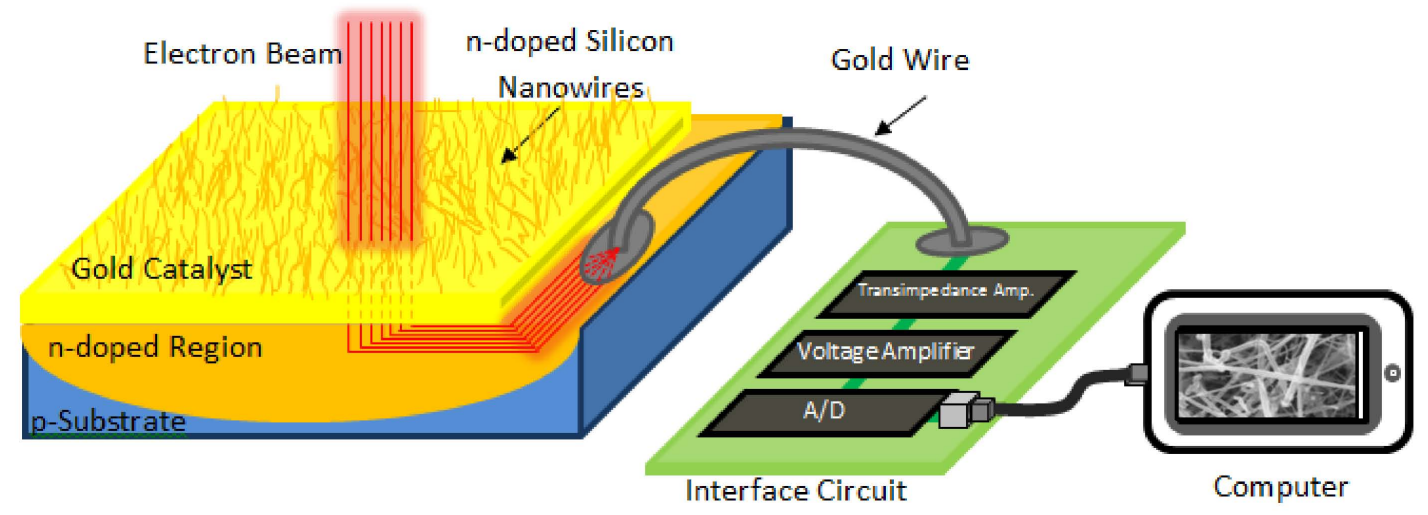

Figure2. Silicon nanowires (SiNWs) on a p-type silicon substrate covered by gold layer as a electron detection system.
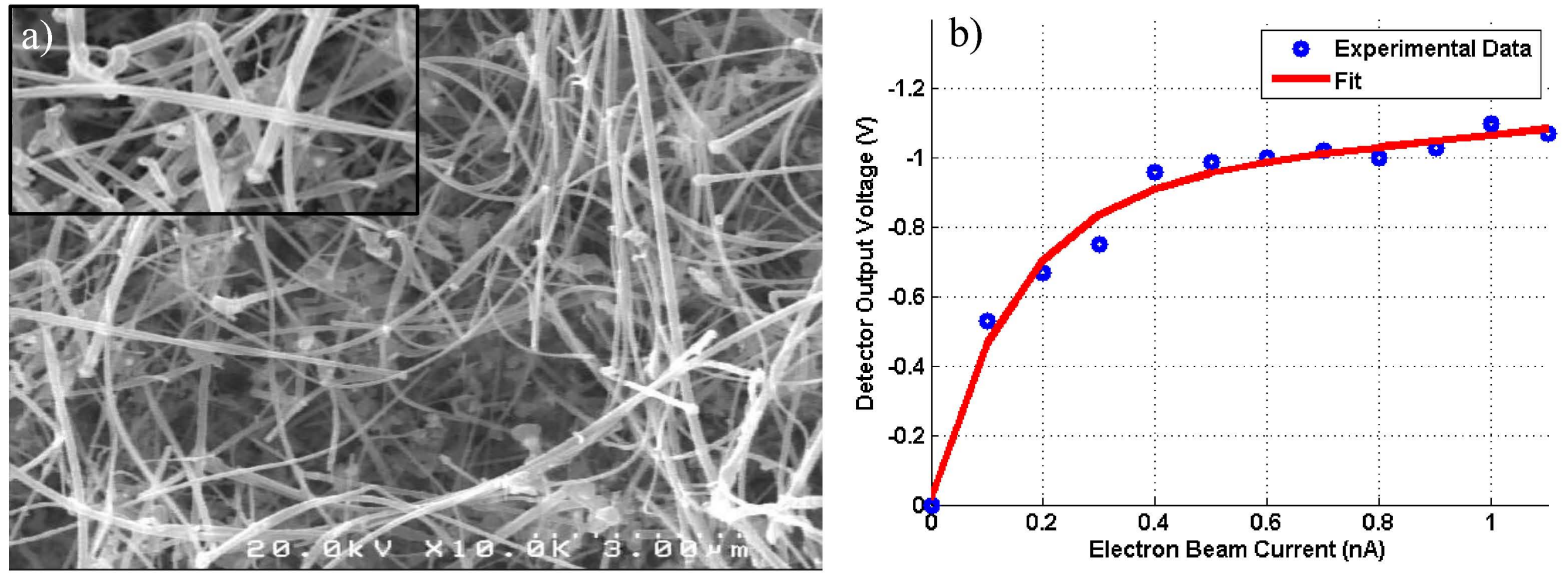

Figure3. a) The SiNWs act as nm electron sensitive conductive wires, b) detector output voltage versus electron beam current. 